\title{
Symmetry, Singularities and Integrability in Complex Dynamics III: Approximate Symmetries and Invariants
}

\author{
$P G L L E A C H^{\dagger^{1} \dagger^{2}}, S M O Y O{ }^{\dagger^{2}}, S$ COTSAKIS ${\dagger^{1} \dagger^{2}}^{2}$ and $R$ L LEMMER ${\dagger^{1} \dagger^{3}}^{2}$ \\ $\dagger^{1}$ Laboratory for Geometry, Dynamical Systems and Cosmology (GEODYSYC) \\ Department of Mathematics, University of the Aegean, Karlovassi 83 200, Greece \\ $\dagger^{2}$ School of Mathematical and Statistical Sciences, University of Natal \\ Durban 4041, Republic of South Africa \\ $\dagger^{3}$ Citadel Investment Services, Pretoria, Republic of South Africa \\ Received July 1, 2000; Revised August 17, 2000; Accepted August 21, 2000
}

\begin{abstract}
The different natures of approximate symmetries and their corresponding first integrals/invariants are delineated in the contexts of both Lie symmetries of ordinary differential equations and Noether symmetries of the Action Integral. Particular note is taken of the effect of taking higher orders of the perturbation parameter. Approximate symmetries of approximate first integrals/invariants and the problems of calculating them using the Lie method are considered.
\end{abstract}

\section{Introduction}

According to Gazizov [6] the first publication devoted to approximate symmetries was by Baikov et. al. [1] (see also [2]). The concept of an approximate symmetry goes back to at least the late seventies [12] when the observation was made that, since the differential equations which arise in mathematical modelling are invariably approximate, one should in fact be considering approximate symmetries. The observation may have been a consequence of a number of papers on applications in Cosmology in the sixties and seventies (cf. [13, 14, 21, 26, 27]). These applications continue [28]. More recently in the context of differential equations the concept was regarded as sufficiently important for Head [10] to add a procedure for the calculation of approximate symmetries to his Program LIE in 1997. The procedure is for computations to the first order in the perturbation parameter $\epsilon$, but the program is easily adapted for the computation to be made to any order (subject to the capacity of the machine).

We recall that a differential equation has a Lie symmetry if there exists an infinitesimal transformation which leaves the differential equation unchanged. The infinitesimal transformation

$$
\begin{aligned}
& \bar{x}=x+\alpha \xi, \\
& \bar{y}=y+\alpha \eta,
\end{aligned}
$$


where $\alpha$ is the infinitesimal parameter, $x$ is the independent variable and $y$ the dependent variable (the extension to more than one of each is simple enough in principle), can be written as

$$
\bar{x}=(1+\alpha G) x, \quad \bar{y}=(1+\alpha G) y,
$$

where $G$ is the differential operator

$$
G=\xi \partial_{x}+\eta \partial_{y}
$$

To deal with differential equations the operator $G$ must be extended to give the infinitesimal transformations in the derivatives induced by the infinitesimal transformations in the independent and dependent variables. The $n$th extension of $G$, which deals with all derivatives up to the $n$th derivative, is [20]

$$
G^{[n]}=G+\sum_{j=1}^{n} \eta^{[j]} \partial_{y^{(j)}},
$$

where

$$
\begin{aligned}
& \eta^{[k]}=\frac{\mathrm{d}}{\mathrm{d} x} \eta^{[k-1]}-y^{(k)} \frac{\mathrm{d} \xi}{\mathrm{d} x}, \quad k=1, n, \\
& \eta^{[0]}=\eta .
\end{aligned}
$$

An $n$th order differential equation,

$$
E\left(x, y, y^{\prime}, \ldots, y^{(n)}\right)=0,
$$

is invariant under the infinitesimal transformation generated by $G$ if

$$
G^{[n]} E_{\left.\right|_{E=0}}=0 .
$$

Each differential operator $G$, with this property is called a symmetry of the differential equation and the aggregation of all such symmetries forms the Lie algebra of the differential equation. A first integral/invariant, $I\left(x, y, y^{\prime}, \ldots, y^{(n-1)}\right)$, is associated with a symmetry $G$ if

$$
G^{[n-1]} I=0 \quad \text { and } \quad \frac{\mathrm{d} I}{\mathrm{~d} x}=0 .
$$

Instead of a differential equation one can consider the Action Integral as the object to maintain its invariance under an infinitesimal transformation. In this case we have Noether's Theorem 23] which states that $G$ is a Noether symmetry of a Lagrangian, $L\left(x, y, y^{\prime}, \ldots, y^{(n)}\right)$, if there exists a function $f$, such that

$$
f^{\prime}=G^{[n]} L+\xi^{\prime} L .
$$

The existence of the symmetry is independent of the Euler-Lagrange equation. When the Variational Principle is invoked to give the Euler-Lagrange equations, (1.9) can be manipulated to give the first integral/invariant

$$
I=f-\left[\xi L+\left(\eta_{i}-y_{i}^{\prime} \xi\right)\right] \frac{\partial L}{\partial y_{i}^{\prime}} .
$$


All of these considerations have been made without reference to the nature of the coefficient functions $\xi$ and $\eta$. In Lie's original work on the symmetries of differential equations he considered both point [17] and contact transformations [18] while Noether used generalized transformations ab initio. There is in fact a priori no restriction on the functional dependence of the coefficient functions and increasingly use is made of generalized and nonlocal symmetries to expand the classes of problems which can be solved. If algebraic properties are the primary consideration, it is better to stay with point or contact symmetries, otherwise there is no need for constraint. In the end the choice of type of infinitesimal transformation to be used is determined by a utilitarianism appropriate to the occasion [8].

No matter the type of infinitesimal transformation under consideration, it is always possible to introduce the approximate form of such a transformation if the differential equation under investigation contains a small constant so that the equation can be regarded as a perturbation of the equation with the constant set at zero. Suppose that the differential equation is

$$
E\left(x, y, y^{\prime}, \ldots, y^{(n)}, \epsilon\right)=0
$$

where $\epsilon$ is the small constant and should not be confused with the $\alpha$ used to denote the infinitesimal parameter in (1.1) and (1.2). The generator of an approximate infinitesimal transformation of order $k$ can be written as

$$
G=\sum_{i=0}^{k} \epsilon^{i} G_{i}
$$

where

$$
\frac{\partial G_{i}}{\partial \epsilon}=0
$$

The generator (1.12) will be an approximate symmetry of (1.11) if

$$
G^{[n]} E_{\mid E=0}=O\left(\epsilon^{k+1}\right) \text {. }
$$

We note that, when $n=0$ in (1.11), we have the case of a function and the definition of an invariant associated with a symmetry group is generalized to that of an approximate invariant or, more correctly, an invariant function of an approximate symmetry group. (In the language of jet bundles, cf. Cotsakis, Leach \& Pantazi [ 4 , one is extending the "approximate" vector field from one on the manifold - in the case of a function - to that on the $n^{t h}$ jet bundle of the total space. A differential equation is then a variety living in the $n^{\text {th }}$ jet bundle and in our case we may reinterpret this as the unperturbed entity of the $\epsilon$-perturbed family of varieties related to the approximate symmetries.)

Noether's Theorem can be adjusted as follows. We consider transformations generated by symmetries of the form (1.12) so that (1.2) becomes, to order $k$ in $\epsilon$,

$$
\bar{x}=x+\alpha \sum_{i=0}^{k} \epsilon^{i} \xi_{i}, \quad \bar{y}=y+\alpha \sum_{i=0}^{k} \epsilon^{i} \eta_{i},
$$

where

$$
G_{i}=\xi_{i} \partial_{x}+\eta_{i} \partial_{y}, \quad \frac{\partial \xi_{i}}{\partial \epsilon}=0=\frac{\partial \eta_{i}}{\partial \epsilon}, \quad i=0, k .
$$


From Noether's theorem we have that $(1.12)$ is an approximate Noether symmetry of the action integral

$$
A=\int_{x_{0}}^{x_{1}} L\left(x, y, y^{\prime}, \alpha\right) \mathrm{d} x
$$

if it leaves the action integral (1.17) unchanged, i.e.

$$
\int_{\bar{x}_{0}}^{\bar{x}_{1}} L\left(\bar{x}, \bar{y}, \bar{y}^{\prime}, \alpha\right) \mathrm{d} x=\int_{x_{0}}^{x_{1}} L\left(x, y, y^{\prime}, \alpha\right) \mathrm{d} x+\alpha F+O\left(\epsilon^{k+1}\right),
$$

where $\alpha F$ is the infinitesimal contribution from the boundary terms and, naturally, is written in terms of an expansion in $\epsilon$ as

$$
\alpha F=\alpha \sum_{i=0}^{k} \epsilon^{i} F_{i}, \quad \frac{\partial F_{i}}{\partial \epsilon}=0=\frac{\partial F_{i}}{\partial \alpha} .
$$

The use of (1.15) in (1.18) up to order $\epsilon^{k}$ leads to the Killing-type equation [25]

$$
\begin{aligned}
\sum_{i=0}^{k} \epsilon^{i} F_{i}^{\prime}= & \sum_{i=0}^{k} \epsilon^{i}\left\{\xi_{i} \frac{\partial L_{0}}{\partial x}+\eta_{i} \frac{\partial L_{0}}{\partial y}+\left(\eta_{i}^{\prime}-y^{\prime} \xi_{i}^{\prime}\right) \frac{\partial L_{0}}{\partial y^{\prime}}+\xi_{i}^{\prime} L_{0}\right\} \\
& +\sum_{i=1}^{k} \epsilon^{i}\left\{\xi_{i} \frac{\partial L_{1}}{\partial x}+\eta_{i} \frac{\partial L_{1}}{\partial y}+\left(\eta_{i}^{\prime}-y^{\prime} \xi_{i}^{\prime}\right) \frac{\partial L_{1}}{\partial y^{\prime}}+\xi_{i}^{\prime} L_{1}\right\}+O\left(\epsilon^{k+1}\right),
\end{aligned}
$$

where prime denotes total differentiation with respect to $x$, we have written $L=L_{0}+\epsilon L_{1}$ in an obvious notation and all terms of order greater than $\epsilon^{k}$ are neglected. Hence (1.16) is an approximate Noether symmetry of (1.17) if the Killing type equation (1.20) is satisfied.

Nothing has been said about the dependence of the functions $\xi_{i}, \eta_{i}$ and $f_{i}$ apart from their lack of dependence upon $\epsilon$. If one seeks approximate point transformations, these functions depend on $x$ and $y$ only. Otherwise the functions can be allowed to depend on $x, y$ and derivatives of $y$. Indeed there is no reason why one should not contemplate nonlocal approximate symmetries. A theory of approximate Noether symmetries based on the concept of approximate infinitesimal transformations can be found in [9]. However, that theory is limited to the first order in $\epsilon$ only and does not lead to the results presented in this paper.

The rest of this paper, which is the third in a series [15, 16] devoted to the investigation of the connections between the three main topics in dynamics, viz symmetry, singularities and integrability, topics which superficially are unrelated in their mathematics and yet are intimately intertwined, is organized as follows. In the next section we examine three simple second order ordinary differential equations for approximate symmetries. The equations are

(i) the simple harmonic oscillator treated as a perturbation of the free particle,

(ii) the Ermakov-Pinney equation treated as a perturbation of the equation for the simple harmonic oscillator and

(iii) an autonomous Emden-Fowler equation of order two considered as a perturbation of the free particle equation. 
Each of these equations illustrates certain aspects of the properties of approximate symmetries. We have chosen simple equations so that the properties of the symmetries will not be obscured by complexity of the equations. In $\S 3$ we consider the properties of approximate Noether symmetries applied to the Lagrangians of the differential equations above. In $\S 4$ we examine some of the problems which arise in the determination of approximate first integrals/invariants using the Lie method and the approximate symmetries associated with them and in $\S 5$ we present our observations and conclusions.

\section{Approximate Lie symmetries: Three case studies}

\subsection{The simple harmonic oscillator}

We write the differential equation as

$$
\ddot{x}+\epsilon x=0,
$$

where $\epsilon$ is a small parameter. Naturally this is somewhat artificial since any value can be used as the coefficient of $x$ by the simple expedient of rescaling time. We recall that the number of exact symmetries is eight no matter the value of $\epsilon$. The calculation of the symmetries is straightforward and we list the approximate symmetries at $O(\epsilon), O\left(\epsilon^{2}\right)$ and $O\left(\epsilon^{3}\right)$. They are

$O(\epsilon)$ :

$$
\begin{array}{ll}
G_{1}=x \partial_{x}, & G_{2}=\epsilon\left(2 t \partial_{t}+x \partial_{x}\right), \\
G_{3}=\left(1-\frac{1}{2} \epsilon t^{2}\right) \partial_{x}, & G_{4}=\left(t-\frac{1}{3 !} \epsilon t^{3}\right) \partial_{x}, \\
G_{5}=x\left(t-\frac{1}{3 !} \epsilon t^{3}\right) \partial_{t}+x^{2}\left(1-\frac{1}{2 !} \epsilon t^{2}\right) \partial_{x}, & G_{6}=\left(t^{2}-\frac{1}{3} \epsilon t^{4}\right) \partial_{t}+x\left(t-\frac{2}{3} t^{3} \epsilon\right) \partial_{x}, \\
G_{7}=x\left(1-\frac{1}{2} \epsilon t^{2}\right) \partial_{t}-t x^{2} \epsilon \partial_{x}, & G_{8}=\epsilon x \partial_{t}, \\
G_{9}=\left(1-2 \epsilon t^{2}\right) \partial_{t}-2 t x \epsilon \partial_{x}, & G_{10}=\left(t-\frac{2}{3} \epsilon t^{3}\right) \partial_{t}+x\left(1-t^{2}\right) \partial_{x}, \\
G_{11}=\epsilon \partial_{t}, & G_{12}=\epsilon \partial_{x}, \\
G_{13}=\epsilon t \partial_{t}, & G_{14}=\epsilon t \partial_{x}, \\
G_{15} & =\epsilon\left(t x \partial_{t}+x^{2} \partial_{x}\right), \\
G_{16} & =\epsilon\left(t^{2} \partial_{t}+t x \partial_{x}\right) ;
\end{array}
$$

$O\left(\epsilon^{2}\right):$

$$
\begin{aligned}
G_{1} & =x \partial_{x}, & G_{2} & =\epsilon^{2}\left(2 t \partial_{t}+x \partial_{x}\right), \\
G_{3} & =\left(1-\frac{1}{2} \epsilon t^{2}+\frac{1}{4 !} \epsilon^{2} t^{4}\right) \partial_{x}, & G_{4} & =\left(t-\frac{1}{3 !} \epsilon t^{3}+\frac{1}{5 !} \epsilon^{2} t^{5}\right) \partial_{x},
\end{aligned}
$$




$$
\begin{aligned}
& G_{5}=\epsilon\left[x\left(t-\frac{1}{3 !} \epsilon t^{3}\right) \partial_{t}+x^{2}\left(1-\frac{1}{2 !} \epsilon t^{2}\right) \partial_{x}\right], \\
& G_{6}=\left(t^{2}-\frac{1}{3} \epsilon t^{4}+\frac{2}{45} \epsilon^{2} t^{6}\right) \partial_{t}+x\left(t-\frac{2}{3} \epsilon t^{3}+\frac{2}{15} \epsilon^{2} t^{5}\right) \partial_{x} \\
& G_{7}=x\left(1-\frac{1}{2} \epsilon t^{2}+\frac{1}{4 !} \epsilon^{2} t^{4}\right) \partial_{t}-x^{2}\left(\epsilon t-\frac{1}{3 !} \epsilon^{2} t^{3}\right) \partial_{x} \\
& G_{8}=\epsilon\left[x\left(1-\frac{1}{2} \epsilon t^{2}\right) \partial_{t}-\epsilon t x^{2} \partial_{x}\right] \text {, } \\
& G_{9}=\left(1-2 \epsilon t^{2}+\frac{2}{3} \epsilon^{2} t^{4}\right) \partial_{t}-x\left(2 \epsilon t-\frac{4}{3} \epsilon^{2} t^{3}\right) \partial_{x}, \\
& G_{10}=\epsilon\left[\left(t-\frac{2}{3} \epsilon t^{3}\right) \partial_{t}+x\left(1-t^{2}\right) \partial_{x}\right], \quad G_{11}=\epsilon^{2} \partial_{t}, \\
& G_{12}=\epsilon\left(1-\frac{1}{2} \epsilon t^{2}\right) \partial_{x}, \quad G_{13}=\left(\epsilon t-\frac{2}{3} \epsilon^{2} t^{3}\right) \partial_{t}-\epsilon^{2} t^{2} x \partial_{x} \\
& G_{14}=\epsilon\left(t-\frac{1}{3 !} \epsilon t^{2}\right) \partial_{x}, \quad G_{15}=\epsilon^{2}\left(t x \partial_{t}+x^{2} \partial_{x}\right), \\
& G_{16}=\epsilon^{2}\left(t^{2} \partial_{t}+t x \partial_{x}\right) \text {, } \\
& G_{17}=\epsilon\left(\frac{2}{3} t^{3}-\frac{2}{15} \epsilon t^{5}\right) \partial_{t}+\epsilon x\left(t^{2}-\frac{1}{3} \epsilon t^{4}\right) \partial_{x} \\
& G_{18}=x\left(t-\frac{1}{3 !} \epsilon t^{3}+\frac{1}{5 !} \epsilon^{2} t^{5}\right) \partial_{t}+x^{2}\left(1-\frac{1}{2} \epsilon t^{2}+\frac{1}{4 !} \epsilon^{2} t^{4}\right) \partial_{x}, \\
& G_{19}=\epsilon^{2} \partial_{x}, \quad G_{20}=\epsilon^{2} x \partial_{t}+\epsilon x \partial_{x}, \\
& G_{21}=\epsilon^{2} t \partial_{t}, \quad G_{22}=\epsilon^{2} t \partial_{x}, \\
& G_{23}=\epsilon\left[\left(1-2 \epsilon t^{2}\right) \partial_{t}-2 \epsilon t x \partial_{x}\right], \quad G_{24}=\epsilon\left[\left(1-2 \epsilon t^{2}\right) \partial_{t}-2 \epsilon t x \partial_{x}\right] ;
\end{aligned}
$$

$O\left(\epsilon^{3}\right)$ :

$$
\begin{aligned}
& G_{1}=x \partial_{x}, \\
& G_{3}=\left(1-\frac{1}{2} \epsilon t^{2}+\frac{1}{4 !} \epsilon^{2} t^{4}-\frac{1}{6 !} \epsilon^{3} t^{6}\right) \partial_{x}, \quad G_{4}=\left(t-\frac{1}{3 !} \epsilon t^{3}+\frac{1}{5 !} \epsilon^{2} t^{5}-\frac{1}{7 !} \epsilon^{3} t^{7}\right) \partial_{x}, \\
& G_{5}=\epsilon^{2}\left[x\left(t-\frac{1}{3 !} \epsilon t^{3}\right) \partial_{t}+x^{2}\left(1-\frac{1}{2 !} \epsilon t^{2}\right) \partial_{x}\right], \\
& G_{6}=\epsilon\left(t^{2}-\frac{1}{3} \epsilon t^{4}+\frac{2}{45} \epsilon^{2} t^{6}\right) \partial_{t}+\epsilon x\left(t-\frac{2}{3} \epsilon t^{3}+\frac{2}{15} \epsilon^{2} t^{5}\right) \partial_{x}, \\
& G_{7}=x\left(1-\frac{1}{2 !} \epsilon t^{2}+\frac{1}{4 !} \epsilon^{2} t^{4}-\frac{1}{6 !} \epsilon^{3} t^{6}\right) \partial_{t}-x^{2}\left(\epsilon t-\frac{1}{3 !} \epsilon^{2} t^{3}+\frac{1}{5 !} \epsilon^{3} t^{5}\right) \partial_{x}, \\
& G_{8}=\epsilon^{2}\left[x\left(1-\frac{1}{2} \epsilon t^{2}\right) \partial_{t}-x^{2} \epsilon t \partial_{x}\right],
\end{aligned}
$$




$$
\begin{aligned}
& G_{9}=\left(1-2 \epsilon t^{2}+\frac{2}{3} \epsilon^{2} t^{4}-\frac{4}{45} \epsilon^{3} t^{6}\right) \partial_{t}-\left(2 x \epsilon t-\frac{4}{3} x \epsilon^{2} t^{3}+\frac{4}{15} x \epsilon^{3} t^{5}\right) \partial_{x}, \\
& G_{10}=\left(t^{2}-\frac{1}{3} \epsilon t^{4}+\frac{2}{45} \epsilon^{2} t^{6}-\frac{1}{315} \epsilon^{3} t^{8}\right) \partial_{t}+\left(x^{2}-\frac{1}{2 !} x^{2} \epsilon t^{2}+\frac{1}{4 !} x^{2} \epsilon^{2} t^{4}-\frac{1}{6 !} x^{2} \epsilon^{3} t^{6}\right) \partial_{x}, \\
& G_{11}=\epsilon^{3} \partial_{t} \\
& G_{12}=\epsilon\left(1-\frac{1}{2} \epsilon t^{2}+\frac{1}{4 !} \epsilon^{2} t^{4}\right) \partial_{x} \\
& G_{13}=\left(2 \epsilon^{2} t-\frac{4}{3} \epsilon^{3} t^{3}\right) \partial_{t}-2 x \epsilon^{3} t^{2} \partial_{x}, \quad G_{14}=\epsilon^{2}\left(t-\frac{1}{3 !} \epsilon t^{3}\right) \partial_{x}, \\
& G_{15}=\epsilon^{3}\left(t x \partial_{t}+x^{2} \partial_{x}\right), \quad G_{16}=\epsilon^{3}\left(t^{2} \partial_{t}+t x \partial_{x}\right), \\
& G_{17}=\left(x t-\frac{1}{3 !} x \epsilon t^{3}+\frac{1}{5 !} x \epsilon^{2} t^{5}-\frac{1}{7 !} x \epsilon^{3} t^{7}\right) \partial_{t}+\left(x t-\frac{2}{3} x \epsilon t^{3}+\frac{2}{15} x \epsilon^{2} t^{5}-\frac{4}{315} x \epsilon^{3} t^{7}\right) \partial_{x}, \\
& G_{18}=\left(2 \epsilon t-\frac{4}{3} \epsilon^{2} t^{3}+\frac{4}{15} \epsilon^{3} t^{5}\right) \partial_{t}+\left(\epsilon x+\frac{2}{3} x \epsilon^{3} t^{4}-2 x t^{2} \epsilon^{2}\right) \partial_{x}, \\
& G_{19}=\epsilon^{3} \partial_{x} \\
& G_{20}=\epsilon^{2}\left(1-\frac{1}{2} \epsilon t^{2}\right) \partial_{x}, \\
& G_{21}=\epsilon^{3} t \partial_{t}, \\
& G_{22}=\epsilon^{3} t \partial_{x} \\
& G_{23}=\left(\epsilon^{2}-2 \epsilon^{3} t^{2}\right) \partial_{t}-2 x \epsilon^{3} t \partial_{x}, \quad G_{24}=\left(2 \epsilon^{2} t-\frac{4}{3} \epsilon^{3} t^{3}\right) \partial_{t}+\left(x \epsilon^{2}-2 x t^{2} \epsilon^{3}\right) \partial_{x}, \\
& G_{25}=\left(2 \epsilon t-\frac{4}{3} \epsilon^{2} t^{3}+\frac{4}{15} \epsilon^{3} t^{5}\right) \partial_{t}-\left(2 x \epsilon^{2} t^{2}-\frac{2}{3} x \epsilon^{3} t^{4}\right) \partial_{x}, \\
& G_{26}=\left(t-\frac{2}{3} \epsilon t^{3}+\frac{2}{15} \epsilon^{2} t^{5}-\frac{4}{315} \epsilon^{3} t^{7}\right) \partial_{t}+\left(\frac{1}{3} x \epsilon^{2} t^{4}-x \epsilon t^{2}-\frac{2}{45} x \epsilon^{3} t^{6}\right) \partial_{x}, \\
& G_{27}=\left(\epsilon-2 \epsilon^{2} t^{2}+\frac{2}{3} \epsilon^{3} t^{4}\right) \partial_{t}-\left(2 x \epsilon^{2} t-\frac{4}{3} x \epsilon^{3} t^{3}\right) \partial_{x}, \\
& G_{28}=\left(x \epsilon-\frac{1}{2} x \epsilon^{2} t^{2}+\frac{1}{4 !} x \epsilon^{3} t^{4}\right) \partial_{t}-\left(x^{2} \epsilon^{2} t-\frac{1}{3 !} x^{2} \epsilon^{3} t^{3}\right) \partial_{x}, \\
& G_{29}=\left(\epsilon^{2} t^{2}-\frac{1}{3} \epsilon^{3} t^{4}\right) \partial_{t}+\left(x \epsilon^{2} t-\frac{2}{3} x \epsilon^{3} t^{3}\right) \partial_{x}, \\
& G_{30}=\left(\epsilon t-\frac{1}{3 !} \epsilon^{2} t^{3}+\frac{1}{5 !} \epsilon^{3} t^{5}\right) \partial_{x}, \\
& G_{31}=\left(x \epsilon t-\frac{1}{3 !} x \epsilon^{2} t^{3}+\frac{1}{5 !} x \epsilon^{3} t^{5}\right) \partial_{t}+\left(x^{2} \epsilon-\frac{1}{2 !} x^{2} \epsilon^{2} t^{2}+\frac{1}{4 !} x^{2} \epsilon^{3} t^{4}\right) \partial_{x}, \\
& G_{32}=\epsilon^{3} x \partial_{t}
\end{aligned}
$$

We observe that there are sixteen approximate symmetries at $O(\epsilon)$, twenty-four at $O\left(\epsilon^{2}\right)$ and thirty-two at $O\left(\epsilon^{3}\right)$. On closer inspection we see that there are different categories of approximate symmetry which would not become apparent if we worked only to $O(\epsilon)$. The homogeneity symmetry, $x \partial / \partial x$, which is common to both perturbed and unperturbed equations occurs at all orders of $\epsilon$. Other symmetries occur at levels of in- 
creasing approximation, for example $G_{3}$, in which one sees the rôle of the perturbation parameter bringing additional terms of a MacLaurin expansion. We recognise the initial terms of the expected sine and cosine series. Then also we see, again with the example of $G_{3}$ at $O(\epsilon)$, that $G_{12}$ is simply $\epsilon$ times the first order $G_{3}$. Hence $G_{3}$ is a true approximate symmetry in contrast with, say, $G_{12}$ which is simply a truncation symmetry. At $O(\epsilon)$ one cannot distinguish between the natures of the two types of symmetry. Consequently we can recognise three types of "approximate" symmetry. The first category comprises those symmetries which are common to both perturbed and unperturbed equations and which occur at all orders of the perturbation parameter. The second class are those symmetries which are truely approximate as one can see the increasing level of approximation with increasing powers of $\epsilon$. They also occur at all levels of approximation. The final category are those which exist by virtue of the power at which the approximation was truncated. They can also have expansions of a form similar to the second category, but are distinguished by never having a term which is independent of $\epsilon$. The number of approximate symmetries increases by eight with each additional order of approximation.

\subsection{The Ermakov-Pinney equation}

The Ermakov-Pinney equation [5, 24] is one of those equations which appears in divers areas and is intimately associated with the $\operatorname{sl}(2, R)$ subalgebra which is the characteristic of all scalar $n$th order ordinary differential equations of maximal symmetry [20]. We write the equation as

$$
\ddot{x}+\omega^{2} x=\frac{\epsilon}{x^{3}}
$$

which has the implication of a perturbation from the equation for a simple harmonic oscillator. We note that both $\omega$ and $\epsilon$ can be scaled to any numbers by rescaling of the variables $x$ and $t$. The Ermakov-Pinney equation has three exact symmetries so that here we are looking at the effect of a decrease in exact symmetries from eight to three. Again we list the approximate symmetries at $O(\epsilon), O\left(\epsilon^{2}\right)$ and $O\left(\epsilon^{3}\right)$. They are listed in (2.6).

The pattern in (2.6) is perhaps easier to discern than in (2.2), (2.3) and (2.4). The first three approximate symmetries, $G_{1}, G_{2}$ and $G_{3}$, are repeated at $O(\epsilon), O\left(\epsilon^{2}\right)$ and $O\left(\epsilon^{3}\right)$ as the order of the approximation is increased and account for the first twelve approximate symmetries (where appropriate). These symmetries, with the algebra $s l(2, R)$, are the symmetries common to both the equation for the simple harmonic oscillator and that for the Ermakov-Pinney equation. The remaining symmetries are all at the maximum power of $\epsilon$ applicable and so are a consequence of the truncation of the perturbation expansion. They represent the remnants of the homogeneity symmetry $\left(G_{15}\right.$ and the solution symmetries $\left(G_{16}\right.$ and $\left.G_{17}\right)$ of the original simple harmonic oscillator. The number of approximate symmetries increases by three at each additional order of approximation. 


$\begin{array}{lll}O(\epsilon) & O\left(\epsilon^{2}\right) & O\left(\epsilon^{3}\right) \\ G_{1}=\partial_{t} & G_{1}=\partial_{t} & G_{1}=\partial_{t} \\ G_{2}=\sin 2 \omega t \partial_{t} & G_{2}=\sin 2 \omega t \partial_{t} & G_{2}=\sin 2 \omega t \partial_{t}+\omega x \cos 2 \omega t \partial_{x} \\ +\omega x \cos 2 \omega t \partial_{x} & +\omega x \cos 2 \omega t \partial_{x} & \\ G_{3}=\cos 2 \omega t \partial_{t} & G_{3}=\cos 2 \omega t \partial_{t}-\omega x \sin 2 \omega t \partial_{x} & G_{3}=\cos 2 \omega t \partial_{t}-\omega x \sin 2 \omega t \partial_{x} \\ -\omega x \sin 2 \omega t \partial_{x} & & \\ G_{4}=\epsilon \partial_{t} & G_{4}=\epsilon \partial_{t} & G_{4}=\epsilon \partial_{t} \\ G_{5}=\epsilon \sin 2 \omega t \partial_{t} & G_{5}=\epsilon\left[\sin 2 \omega t \partial_{t}+\omega x \cos 2 \omega t \partial_{x}\right] & G_{5}=\epsilon\left[\sin 2 \omega t \partial_{t}+\omega x \cos 2 \omega t \partial_{x}\right] \\ +\epsilon \omega x \cos 2 \omega t \partial_{x} & & \\ G_{6}=\epsilon \cos 2 \omega t \partial_{t} & G_{6}=\epsilon\left[\cos 2 \omega t \partial_{t}-\omega x \sin 2 \omega t \partial_{x}\right] & G_{6}=\epsilon\left[\cos 2 \omega t \partial_{t}-\omega x \sin 2 \omega t \partial_{x}\right] \\ -\epsilon \omega x \sin 2 \omega t \partial_{x} & & \\ & G_{7}=\epsilon^{2} \partial_{t} & G_{7}=\epsilon^{2} \partial_{t} \\ & G_{8}=\epsilon^{2} \sin 2 \omega t \partial_{t}+\epsilon^{2} \omega x \cos 2 \omega t \partial_{x} & G_{8}=\epsilon^{2}\left[\sin 2 \omega t \partial_{t}+\omega x \cos 2 \omega t \partial_{x}\right] \\ & G_{9}=\epsilon^{2}\left[\cos 2 \omega t \partial_{t}-\omega x \sin 2 \omega t \partial_{x}\right] & G_{9}=\epsilon^{2}\left[\cos 2 \omega t \partial_{t}-\omega x \sin 2 \omega t \partial_{x}\right] \\ & & G_{10}=\epsilon^{3} \partial_{t} \\ G_{13}=\epsilon x \partial_{x} & G_{13}=\epsilon^{2} x \partial_{x} & G_{11}=\epsilon^{3}\left[\sin 2 \omega t \partial_{t}+\omega x \cos 2 \omega t \partial_{x}\right] \\ G_{14}=\epsilon \sin \omega t \partial_{x} & G_{14}=\epsilon^{2} \sin \omega t \partial_{x} & \left.G_{13}=\epsilon^{3} x \partial_{x} 2 \omega t \partial_{t}-\omega x \sin 2 \omega t \partial_{x}\right] \\ G_{15}=\epsilon \cos \omega t \partial_{x} & G_{15}=\epsilon^{2} \cos \omega t \partial_{x} & G_{14}=\epsilon^{3} \sin \omega t \partial_{x} \\ G_{16}=\epsilon x \sin \omega t \partial_{t} & G_{16}=\epsilon^{2}\left[x \sin \omega t \partial_{t}+\omega x^{2} \cos \omega t \partial_{x}\right] & G_{16}=\epsilon^{3}\left[x \cos \omega t \partial_{x}\right. \\ +\epsilon \omega x^{2} \cos \omega t \partial_{x} & & \\ \left.G_{17}=\epsilon x \cos \omega t \partial_{t}+\omega x^{2} \cos \omega t \partial_{x}\right] \\ -\epsilon \omega x^{2} \sin \omega t \partial_{x} & G_{17}=\epsilon^{2}\left[x \cos \omega t \partial_{t}-\omega x^{2} \sin \omega t \partial_{x}\right] & G_{17}=\epsilon^{3}\left[x \cos \omega t \partial_{t}-\omega x^{2} \sin \omega t \partial_{x}\right] .\end{array}$

\subsection{Emden-Fowler equation}

We consider the Emden-Fowler equation

$$
\ddot{x}=\epsilon x^{2},
$$

i.e. as a perturbation of the free particle equation. Again the parameter can be set at any value by rescaling. We find the approximate symmetries as are listed in (2.9).

The symmetries $G_{1}$ and $G_{2}$ are repeated at higher orders of approximation and so are common to both equations. In the normal listing of the Lie point symmetries of the free particle one would have the two symmetries

$$
X_{1}=x \partial_{x} \quad \text { and } \quad X_{2}=t \partial_{t}+\frac{1}{2} x \partial_{x},
$$

the homogeneity symmetry and the middle element of the $s l(2, R)$ triplet in the form appropriate to a second order ordinary differential equation [20], but any linear combination of $X_{1}$ and $X_{2}$ is also a symmetry. The combination which gives $G_{2}$ is common to both equations and so is maintained at all orders of $\epsilon$ whereas the combinations which give $G_{9}$ and $G_{12}$ are not inherited, but are simply a consequence of the order of the approximate symmetry. We also observe that the perturbations of $G_{1}$ occur to the order of $\epsilon$ being 
used in the approximate symmetry whereas those of $G_{2}$ are at one order less. This is because the differential equation, (2.7), is used in the calculation of the second approximate symmetry but not the first which simply annihilates the equation.

$\begin{array}{lll}O(\epsilon) & O\left(\epsilon^{2}\right) & O\left(\epsilon^{3}\right) \\ G_{1}=\partial_{t} & G_{1}=\partial_{t} & G_{1}=\partial_{t} \\ G_{2}=t \partial_{t}-2 x \partial_{x} & G_{2}=t \partial_{t}-2 x \partial_{x} & G_{2}=t \partial_{t}-2 x \partial_{x} \\ G_{3}=\epsilon \partial_{t} & G_{3}=\epsilon \partial_{t} & G_{3}=\epsilon \partial_{t} \\ & G_{4}=\epsilon\left[t \partial_{t}-2 x \partial_{x}\right] & G_{4}=\epsilon\left[t \partial_{t}-2 x \partial_{x}\right] \\ & G_{5}=\epsilon^{2} \partial_{t} & G_{5}=\epsilon^{2} \partial_{t} \\ & & G_{6}=\epsilon^{2}\left[t \partial_{t}-2 x \partial_{x}\right] \\ & G_{7}=\epsilon^{3} \partial_{t} \\ G_{8}=\epsilon \partial_{x} & G_{8}=\epsilon^{2} \partial_{x} & G_{8}=\epsilon^{3} \partial_{x} \\ G_{9}=\epsilon t \partial_{t} & G_{9}=\epsilon^{2} t \partial_{t} & G_{9}=\epsilon^{3} t \partial_{t} \\ G_{10}=\epsilon x \partial_{t} & G_{10}=\epsilon^{2} x \partial_{t} & G_{10}=\epsilon^{3} x \partial_{t} \\ G_{11}=\epsilon t \partial_{x} & G_{11}=\epsilon^{2} t \partial_{x} & G_{11}=\epsilon^{3} t \partial_{x} \\ G_{12}=\epsilon x \partial_{x} & G_{12}=\epsilon^{2} x \partial_{x} & G_{12}=\epsilon^{3} x \partial_{x} \\ G_{13}=\epsilon\left[t x \partial_{t}+x^{2} \partial_{x}\right] & G_{13}=\epsilon^{2}\left[t x \partial_{t}+x^{2} \partial_{x}\right] & G_{13}=\epsilon^{3}\left[t x \partial_{t}+x^{2} \partial_{x}\right] \\ G_{14}=\epsilon\left[t^{2} \partial_{t}+t x \partial_{x}\right] & G_{14}=\epsilon^{2}\left[t^{2} \partial_{t}+t x \partial_{x}\right] & G_{14}=\epsilon^{3}\left[t^{2} \partial_{t}+t x \partial_{x}\right] \\ G_{15}=\epsilon t^{4} \partial_{t} & G_{15}=\epsilon\left[\epsilon t^{4} \partial_{t}+\left(6 t+2 \epsilon t^{3} x\right) \partial_{x}\right] & G_{15}=\epsilon^{2}\left[t^{4} \partial_{t}+\left(6 t+2 \epsilon t^{3} x\right) \partial_{x}\right] \\ +\left(6 t+2 \epsilon t^{3} x\right) \partial_{x} & & \\ G_{16}=2 \epsilon t^{3} \partial_{t} & G_{16}=\epsilon\left[2 \epsilon t^{3} \partial_{t}+3\left(1+\epsilon t^{2} x\right) \partial_{x}\right] & G_{16}=\epsilon^{2}\left[2 \epsilon t^{3} \partial_{t}+3\left(1+\epsilon t^{2} x\right) \partial_{x}\right] . \\ +3\left(1+\epsilon t^{2} x\right) \partial_{x} & & \end{array}$

\section{The Noether symmetries and approximate first integrals/invariants}

\subsection{The simple harmonic oscillator}

The simple harmonic oscillator has the Lagrangian

$$
L=\frac{1}{2}\left[\dot{x}^{2}-\epsilon x^{2}\right] .
$$

Given the observations made in the previous section we know the pattern of the behaviour of the symmetries and so we will simply list the results for the first two orders of $\epsilon$. We consider point symmetries only. Because of the direct relationship between symmetry and first integral we list the $G \mathrm{~s}, f_{\mathrm{s}}$ and $I$ s together. The results are given in (3.2) and (3.3).

The only Noether symmetry of the free particle which is inherited by the simple harmonic oscillator is $G_{1}$. Since both Lagrangians have five Noether point symmetries, at $O(\epsilon)$ we see that there are two symmetries due to the inheritance of the exact symmetry $G_{1}$, four approximate symmetries of the free particle which persist as truncation symmetries and four genuine approximation symmetries of the simple harmonic oscillator. We see the beginnings of the MacLaurin expansions for the sine and cosine functions. The behaviour of the integrals reflects these distinctions. At $O\left(\epsilon^{2}\right)$ we gain an extra five symmetries. One of these is due to the inherited symmetry and the other four because the 
four approximate symmetries of the simple harmonic oscillator at order one will have the same behaviour as the inherited symmetry at order two. The distinction between the two classes is that for the inherited symmetry all occurrences are simply multiples of the zeroth order symmetry by powers of $\epsilon$ whereas the basic approximation symmetries increase the degree of approximation with increasing powers of $\epsilon$ and the others are multiple of approximate symmetries at lower order. The truncation symmetries do not multiply, but simply persist at the order of the approximation being used.

To the first order in $\epsilon$ we have

\begin{tabular}{lll}
\hline Symmetry & Gauge function & Invariant \\
\hline$G_{1}=\partial_{t}$ & $f_{1}=0$ & $I_{1}=\frac{1}{2}\left(\dot{x}^{2}+\epsilon x^{2}\right)$ \\
$G_{2}=\epsilon \partial_{t}$ & $f_{2}=0$ & $I_{2}=\frac{1}{2} \epsilon \dot{x}^{2}$ \\
$G_{3}=\left[2 t-\frac{1}{3 !} \epsilon(2 t)^{3}\right] \partial_{t}$ & $f_{3}=-2 \epsilon t x^{2}$ & $I_{3}=\dot{x}(t \dot{x}-x)-\epsilon t\left(x^{2}-2 t x \dot{x}+\frac{2}{3} t^{2} \dot{x}^{2}\right)$ \\
$+x\left[2 t-\frac{1}{2 !} \epsilon(2 t)^{2}\right] \partial_{x}$ & & \\
$G_{4}=\epsilon\left(2 t \partial_{t}+x \partial_{x}\right)$ & $f_{4}=0$ & $I_{4}=\epsilon \dot{x}(t \dot{x}-x)$ \\
$G_{5}=\left(1-\frac{1}{2 !} \epsilon t^{2}\right) \partial_{x}$ & $f_{5}=-\epsilon t x$ & $I_{5}=\left(1-\frac{1}{2 !} \epsilon t^{2}\right) \dot{x}+\epsilon t x$ \\
$G_{6}=\epsilon \partial_{x}$ & $f_{6}=0$ & $I_{6}=\epsilon \dot{x}$ \\
$G_{7}=\left(t-\frac{1}{3 !} \epsilon t^{3}\right) \partial_{x}$ & $f_{7}=\left(1-\frac{1}{2 !} \epsilon t^{2}\right) x$ & $I_{7}=\left(1-\frac{1}{2 !} \epsilon t^{2}\right) x-\left(t-\frac{1}{3 !} \epsilon t^{3}\right) \dot{x}$ \\
$G_{8}=\epsilon t \partial_{x}$ & $f_{8}=\epsilon x$ & $I_{8}=\epsilon(x-t \dot{x})$ \\
$G_{9}=\left[\frac{1}{2 !}(2 t)^{2}-\frac{1}{4 !} \epsilon(2 t)^{4}\right] \partial_{t}$ & $f_{9}=\left(1-\frac{1}{2 !} \epsilon(2 t)^{2}\right) x^{2}$ & $I_{9}=(x-t \dot{x})^{2}-\frac{1}{3} \epsilon t^{2}(3 x-t \dot{x})(x-t \dot{x})$ \\
$+x\left[2 t-\frac{1}{3 !} \epsilon(2 t)^{3}\right] \partial_{x}$ & & \\
$G_{10}=\frac{1}{2 !} \epsilon\left((2 t)^{2} \partial_{t}+x(2 t) \partial_{x}\right)$ & $f_{10}=\epsilon x^{2}$ & $I_{10}=\epsilon(x-t \dot{x})^{2}$ \\
\hline
\end{tabular}

and to the second order in $\epsilon$ we have

\begin{tabular}{lll}
\hline Symmetry & Gauge function & Invariant \\
\hline$G_{1}=\partial_{t}$ & $f_{1}=0$ & $\left.I_{1}=\frac{1}{2} \dot{x}^{2}+\epsilon x^{2}\right)$ \\
$G_{2}=\epsilon \partial_{t}$ & $f_{2}=0$ & $I_{2}=\frac{1}{2} \epsilon\left(\dot{x}^{2}+\epsilon x^{2}\right)$ \\
$G_{3}=\epsilon^{2} \partial_{t}$ & $f_{3}=0$ & $I_{3}=\frac{1}{2} \epsilon \dot{x}^{2}$ \\
$G_{4}=\left[2 t-\frac{1}{3 !} \epsilon(2 t)^{3}+\frac{1}{5} \epsilon^{2}(2 t)^{5}\right] \partial_{t}$ & $f_{4}=-\epsilon x^{2}\left[2 t-\frac{1}{3 !} \epsilon(2 t)^{3}\right]$ & $I_{4}=\dot{x}(t \dot{x}-x)$ \\
$+x\left[2 t-\frac{1}{2 !} \epsilon(2 t)^{2}+\frac{1}{4 !} \epsilon^{2}(2 t)^{4}\right] \partial_{x}$ & & $-\frac{1}{3} \epsilon t\left(3 \dot{x}^{2}-6 t x \dot{x}+2 t^{2} \dot{x}^{2}\right)$ \\
& & $+\frac{2}{15} \epsilon^{2} t^{2}\left(5 x^{2}-5 t x \dot{x}+3 t^{2} \dot{x}^{2}\right)$ \\
$G_{5}=\epsilon\left[2 t-\frac{1}{3 !} \epsilon(2 t)^{3}\right] \partial_{t}$ & $f_{5}=-2 \epsilon^{2} t x^{2}$ & $I_{5}=\epsilon \dot{x}(t \dot{x}-x)$ \\
$+\epsilon x\left[2 t-\frac{1}{2 !} \epsilon(2 t)^{2}\right] \partial_{x}$ & & $-\epsilon^{2} t\left(x^{2}-2 t x \dot{x}+\frac{2}{3} t^{2} \dot{x}^{2}\right)$ \\
$G_{6}=\epsilon^{2}\left(2 t \partial_{t}+x \partial_{x}\right)$ & $I_{6}=\epsilon^{2} \dot{x}(t \dot{x}-x)$ \\
$G_{7}=\left(1-\frac{1}{2 !} \epsilon t^{2}+\frac{1}{4 !} \epsilon^{2} t^{4}\right) \partial_{x}$ & $f_{7}=-\epsilon x\left(t-\frac{1}{3 !} \epsilon t^{3}\right)$ & $I_{7}=\left(1-\frac{1}{2 !} \epsilon t^{2}+\frac{1}{4 !} \epsilon^{2} t^{4}\right) \dot{x}$ \\
& & $+\epsilon x\left(t-\frac{1}{3 !} \epsilon t^{3}\right)$ \\
$G_{8}=\epsilon\left(1-\frac{1}{2 !} \epsilon t^{2}\right) \partial_{x}$ & $f_{5}=-\epsilon^{2} t x$ & $I_{5}=\epsilon\left[\left(1-\frac{1}{2 !} \epsilon t^{2}\right) \dot{x}+\epsilon t x\right]$ \\
$G_{9}=\epsilon^{2} \partial_{x}$ & $f_{9}=0$ & $I_{9}=\epsilon^{2} \dot{x}$ \\
$G_{10}=\left(t-\frac{1}{3 !} \epsilon t^{3}+\frac{1}{5 !} \epsilon^{2} t^{5}\right) \partial_{x}$ & $f_{10}=\left(1-\frac{1}{2 !} \epsilon t^{2}+\frac{1}{4 !} \epsilon^{2} t^{4}\right)$ & $I_{10}=\left(1-\frac{1}{2 !} \epsilon t^{2}+\frac{1}{4} \epsilon^{2} t^{4}\right) x$ \\
$G_{11}=\epsilon\left(t-\frac{1}{3 !} \epsilon t^{3}\right) \partial_{x}$ & $f_{11}=\epsilon\left(1-\frac{1}{2 !} \epsilon t^{2}\right) x$ & $-\left(t-\frac{1}{3 !} \epsilon t^{3}+\frac{1}{5 !} \epsilon^{2} t^{5}\right) \dot{x}$ \\
$G_{12}=\epsilon^{2} t \partial_{x}$ & $I_{11}=\epsilon\left(1-\frac{1}{2 !} \epsilon \dot{t}^{2}\right) x$ \\
$G_{13}=\left[\frac{1}{2 !}(2 t)^{2}-\frac{1}{4 !} \epsilon(2 t)^{4}+\frac{1}{6 !} \epsilon^{2} t^{6}\right] \partial_{t}$ & $f_{13}=\left(1-\frac{1}{2 !} \epsilon(2 t)^{2}\right)^{2}$ & $-\epsilon\left(t-\frac{1}{3 !} \epsilon t^{3} \dot{x}^{2}\right.$ \\
$+x\left[2 t-\frac{1}{3 !} \epsilon(2 t)^{3}+\frac{1}{5 !} \epsilon^{2} t^{5}\right] \partial_{x}$ & $+\frac{1}{4 !} \epsilon^{2}(2 t)^{4} x^{2}$ & $I_{12}=\epsilon^{2}(x-t \dot{x})$ \\
$G_{14}=\epsilon\left(\frac{1}{2 !}(2 t)^{2}-\frac{1}{4 !} \epsilon(2 t)^{4}\right) \partial_{t}$ & $f_{14}=\epsilon\left(1-\frac{1}{2 !} \epsilon(2 t)^{2}\right) x^{2}$ & $\frac{1}{3} \epsilon t^{2}(3 x-t \dot{x})(x-t \dot{x})$ \\
$+\epsilon x\left(2 t-\frac{1}{3 !} \epsilon(2 t)^{3}\right) \dot{\partial}_{x}$ & $I_{14}=\epsilon(x-t \dot{x})^{2}$ \\
$G_{15}=\frac{1}{2 !} \epsilon^{2}\left((2 t)^{2} \partial_{t}+x(2 t) \partial_{x}\right)$ & $f_{15}=\epsilon^{2} x^{2}$ & $-\frac{1}{3} \epsilon^{2} t^{2}(3 x-t \dot{x})(x-t \dot{x})$ \\
\hline
\end{tabular}




\subsection{Ermakov-Pinney system}

The Lagrangian for the Ermakov-Pinney system is

$$
L=\frac{1}{2}\left[\dot{x}^{2}-\omega^{2} x^{2}-\frac{\epsilon}{x^{2}}\right] .
$$

To the first order in $\epsilon$ we have

\begin{tabular}{lll}
\hline Symmetry & Gauge function & Invariant \\
\hline$G_{1}=\partial_{t}$ & $f_{1}=0$ & $I_{1}=\frac{1}{2}\left(\dot{x}^{2}+\omega^{2} x^{2}+\frac{\epsilon}{x^{2}}\right)$ \\
$G_{2}=\epsilon \partial_{t}$ & $f_{2}=0$ & $I_{2}=\frac{1}{2} \epsilon\left(\dot{x}^{2}+\omega^{2} x^{2}\right)$ \\
$G_{3}=\sin 2 \omega t \partial_{t}+\omega x \cos 2 \omega t \partial_{x}$ & $f_{3}=-\omega^{2} x^{2} \sin 2 \omega t$ & $I_{3}=\frac{1}{2}\left(\dot{x}^{2}-\omega^{2} x^{2}+\frac{\epsilon}{x^{2}}\right) \sin 2 \omega t$ \\
& & $-\omega x \dot{x} \cos 2 \omega t$ \\
$G_{4}=\cos 2 \omega t \partial_{t}-\omega x \sin 2 \omega t \partial_{x}$ & $f_{4}=\omega^{2} x^{2} \cos 2 \omega t$ & $I_{4}=\frac{1}{2}\left(\dot{x}^{2}-\omega^{2} x^{2}+\frac{\epsilon}{x^{2}}\right) \cos 2 \omega t$ \\
& & $+\omega x \dot{x} \sin 2 \omega t$ \\
$G_{5}=\epsilon\left[\sin 2 \omega t \partial_{t}+\omega x \cos 2 \omega t \partial_{x}\right]$ & $f_{5}=-\epsilon \omega^{2} x^{2} \sin 2 \omega t$ & $I_{5}=\epsilon \frac{1}{2}\left(\dot{x}^{2}-\omega^{2} x^{2}\right) \sin 2 \omega t$ \\
& & $-\epsilon \omega x \dot{x} \cos 2 \omega t$ \\
$G_{6}=\epsilon\left[\cos 2 \omega t \partial_{t}-\omega x \sin 2 \omega t \partial_{x}\right]$ & $f_{6}=\epsilon \omega^{2} x^{2} \cos 2 \omega t$ & $I_{6}=\epsilon \frac{1}{2}\left(\dot{x}^{2}-\omega^{2} x^{2}\right) \cos 2 \omega t$ \\
& & $+\epsilon \omega x \dot{x} \sin 2 \omega t$ \\
$G_{7}=\epsilon \sin \omega t \partial_{x}$ & $f_{7}=\epsilon \omega x \cos \omega t$ & $I_{7}=\epsilon(\dot{x} \sin \omega t-\omega x \cos \omega t)$ \\
$G_{8}=\epsilon \cos \omega t \partial_{x}$ & $f_{8}=-\epsilon \omega x \sin \omega t$ & $I_{8}=\epsilon(\dot{x} \cos \omega t+\omega x \sin \omega t)$ \\
\hline
\end{tabular}

and to the second order in $\epsilon$

\begin{tabular}{lll}
\hline Symmetry & Gauge function & Invariant \\
\hline$G_{1}=\partial_{t}$ & $f_{1}=0$ & $I_{1}=\frac{1}{2}\left(\dot{x}^{2}+\omega^{2} x^{2}+\frac{\epsilon}{x^{2}}\right)$ \\
$G_{2}=\epsilon \partial_{t}$ & $f_{2}=0$ & $I_{2}=\frac{1}{2} \epsilon\left(\dot{x}^{2}+\omega^{2} x^{2}+\frac{\epsilon}{x^{2}}\right)$ \\
$G_{3}=\epsilon^{2} \partial_{t}$ & $f_{3}=0$ & $I_{3}=\frac{1}{2} \epsilon^{2}\left(\dot{x}^{2}+\omega^{2} x^{2}\right)$ \\
$G_{4}=\sin 2 \omega t \partial_{t}+\omega x \cos 2 \omega t \partial_{x}$ & $f_{4}=-\omega^{2} x^{2} \sin 2 \omega t$ & $I_{4}=\frac{1}{2}\left(\dot{x}^{2}-\omega^{2} x^{2}+\frac{\epsilon}{x^{2}}\right) \sin 2 \omega t$ \\
& & $-\omega x \dot{x} \cos 2 \omega t$ \\
$G_{5}=\cos 2 \omega t \partial_{t}-\omega x \sin 2 \omega t \partial_{x}$ & $f_{5}=\omega^{2} x^{2} \cos 2 \omega t$ & $I_{5}=\frac{1}{2}\left(\dot{x}^{2}-\omega^{2} x^{2}+\frac{\epsilon}{x^{2}}\right) \cos 2 \omega t$ \\
& & $+\omega x \dot{x} \sin 2 \omega t$ \\
$G_{6}=\epsilon\left[\sin 2 \omega t \partial_{t}+\omega x \cos 2 \omega t \partial_{x}\right]$ & $f_{6}=-\epsilon \omega^{2} x^{2} \sin 2 \omega t$ & $I_{6}=\frac{1}{2} \epsilon\left(\dot{x}^{2}-\omega^{2} x^{2}+\frac{\epsilon}{x^{2}}\right) \sin 2 \omega t$ \\
& & $-\epsilon \omega x \dot{x} \cos 2 \omega t$ \\
$G_{7}=\epsilon\left[\cos 2 \omega t \partial_{t}-\omega x \sin 2 \omega t \partial_{x}\right]$ & $f_{7}=\epsilon \omega^{2} x^{2} \cos 2 \omega t$ & $I_{7}=\frac{1}{2} \epsilon\left(\dot{x}^{2}-\omega^{2} x^{2}+\frac{\epsilon}{x^{2}}\right) \cos 2 \omega t$ \\
& & $+\epsilon \omega x \dot{x} \sin 2 \omega t$ \\
$G_{8}=\epsilon^{2} \sin \omega t \partial_{x}$ & $f_{8}=\epsilon^{2} \omega x \cos \omega t$ & $I_{8}=\epsilon^{2}(\dot{x} \sin \omega t-\omega x \cos \omega t)$ \\
$G_{9}=\epsilon^{2} \cos \omega t \partial_{x}$ & $f_{8}=-\epsilon^{2} \omega x \sin \omega t$ & $I_{8}=\epsilon^{2}(\dot{x} \cos \omega t+\omega x \sin \omega t)$ \\
$G_{10}=\epsilon^{2}\left[\sin 2 \omega t \partial_{t}+\omega x \cos 2 \omega t \partial_{x}\right]$ & $f_{10}=-\epsilon^{2} \omega^{2} x^{2} \sin 2 \omega t$ & $I_{10}=\frac{1}{2} \epsilon^{2}\left(\dot{x}^{2}-\omega^{2} x^{2}\right) \sin 2 \omega t$ \\
$G_{11}=\epsilon^{2}\left[\cos 2 \omega t \partial_{t}-\omega x \sin 2 \omega t \partial_{x}\right]$ & $f_{11}=\epsilon \omega^{2} x^{2} \cos 2 \omega t$ & $-\epsilon^{2} \omega x \dot{x} \cos 2 \omega t$ \\
& & $I_{11}=\frac{1}{2} \epsilon^{2}\left(\dot{x}^{2}-\omega^{2} x^{2}\right) \cos 2 \omega t$ \\
\hline
\end{tabular}


We observe that the three symmetries, $G_{1}, G_{2}$ and $G_{3}$, of (2.6) are common Lie and Noether symmetries of the equation for the oscillator and the Ermakov-Pinney equation. They constitute the algebra $\operatorname{sl}(2, R)$ which is the symmetry algebra for the latter and a subalgebra of the $\operatorname{sl}(3, R)$ algebra of the former. The solution symmetries are preserved by the order of truncation. The other three approximate Lie symmetries are not approximate Noether symmetries. The only serious first integrals/invariants correspond to these three symmetries. They are exact and not approximate.

\subsection{Emden-Fowler Lagrangian}

The Lagrangian for the Emden-Fowler equation (2.7) is

$$
L=\frac{1}{2} \dot{x}^{2}+\frac{1}{3} \epsilon x^{3} .
$$

To the first order in $\epsilon$ we have

\begin{tabular}{lll}
\hline Symmetry & Gauge function & Invariant \\
\hline$G_{1}=\partial_{t}$ & $f_{1}=0$ & $I_{1}=\frac{1}{2} \dot{x}^{2}-\frac{1}{3} \epsilon x^{3}$ \\
$G_{2}=\epsilon \partial_{t}$ & $f_{2}=0$ & $I_{2}=\frac{1}{2} \epsilon \dot{x}^{2}$ \\
$G_{3}=\epsilon \partial_{x}$ & $f_{3}=0$ & $I_{3}=\epsilon \dot{x}$ \\
$G_{4}=\epsilon t \partial_{x}$ & $f_{4}=0$ & $I_{4}=\epsilon(x-\dot{x} t)$ \\
$G_{5}=\epsilon\left[2 t \partial_{t}+x \partial_{x}\right]$ & $f_{5}=0$ & $I_{5}=\epsilon \dot{x}(x-\dot{x} t)$ \\
$G_{6}=\epsilon\left[t^{2} \partial_{t}+t x \partial_{x}\right]$ & $f_{6}=\frac{1}{2} \epsilon x^{2}$ & $I_{6}=\frac{1}{2} \epsilon(x-t \dot{x})^{2}$ \\
$G_{7}=\frac{1}{3 !} \epsilon t^{4} \partial_{t}+\left(t+\frac{1}{3} \epsilon t^{3} x\right) \partial_{x}$ & $f_{7}=\frac{1}{2} \epsilon t^{2} x^{2}$ & $I_{7}=t \dot{x}-\epsilon\left(\frac{1}{12} t^{4}-\frac{1}{3} t^{3} x \dot{x}+\frac{1}{2} t^{2} x^{2}\right)$ \\
$G_{8}=\frac{2}{3} \epsilon t^{3} \partial_{t}+\left(1+\epsilon t^{2} x\right) \partial_{x}$ & $f_{8}=\epsilon t x^{2}$ & $I_{8}=\dot{x}-\epsilon\left(t x^{2}-t^{2} x \dot{x}+\frac{1}{3} t^{3} \dot{x}^{2}\right)$ \\
\hline
\end{tabular}

and to the second order in $\epsilon$ we have

\begin{tabular}{lll}
\hline Symmetry & Gauge function & Invariant \\
\hline$G_{1}=\partial_{t}$ & $f_{1}=0$ & $I_{1}=\frac{1}{2} \dot{x}^{2}-\frac{1}{3} \epsilon x^{3}$ \\
$G_{2}=\epsilon \partial_{t}$ & $f_{2}=0$ & $I_{2}=\epsilon\left(\frac{1}{2} \dot{x}^{2}-\frac{1}{3} \epsilon x^{3}\right)$ \\
$G_{3}=\epsilon^{2} \partial_{t}$ & $f_{3}=0$ & $I_{3}=\frac{1}{2} \epsilon^{2} \dot{x}^{2}$ \\
$G_{4}=\epsilon^{2} \partial_{x}$ & $f_{3}=0$ & $I_{4}=\epsilon^{2} \dot{x}$ \\
$G_{5}=\epsilon^{2} t \partial_{x}$ & $f_{5}=0$ & $I_{5}=\epsilon^{2}(x-\dot{x} t)$ \\
$G_{6}=\epsilon^{2}\left[2 t \partial_{t}+x \partial_{x}\right]$ & $f_{6}=0$ & $I_{6}=\epsilon^{2} \dot{x}(x-\dot{x} t)$ \\
$G_{7}=\epsilon^{2}\left[t^{2} \partial_{t}+t x \partial_{x}\right]$ & $f_{7}=\frac{1}{2} \epsilon^{2} x^{2}$ & $I_{7}=\frac{1}{2} \epsilon^{2}(x-t \dot{x})^{2}$ \\
$G_{8}=\epsilon\left[\frac{1}{3 !} \epsilon t^{4} \partial_{t}+\left(t+\frac{1}{3} \epsilon t^{3} x\right) \partial_{x}\right]$ & $f_{8}=\frac{1}{2} \epsilon^{2} t^{2} x^{2}$ & $I_{8}=\epsilon\left[t \dot{x}-\epsilon\left(\frac{1}{12} t^{4}-\frac{1}{3} t^{3} x \dot{x}+\frac{1}{2} t^{2} x^{2}\right)\right]$ \\
$G_{9}=\epsilon\left[\frac{2}{3} \epsilon t^{3} \partial_{t}+\left(1+\epsilon t^{2} x\right) \partial_{x}\right]$ & $f_{9}=\epsilon t x^{2}$ & $\left.I_{9}=\epsilon \dot{x}-\epsilon\left(t x^{2}-t^{2} x \dot{x}+\frac{1}{3} t^{3} \dot{x}^{2}\right)\right]$ \\
\hline
\end{tabular}

The only Noether symmetry which produces a useful integral is $G_{1}$. Had one treated only to $O(\epsilon)$, which is the implication of the one of the few texts giving a treatment of approximate symmetries [11], one may have become enthusiastic about the integrals produced by $G_{7}$ and $G_{8}$. Indeed they appear to be classic examples of a perturbation solution. However, we see from the $O\left(\epsilon^{2}\right)$ results that these symmetries are simply the result of truncation since the order of $\epsilon$ increases with the order of the approximation. We remark that the results for this Lagrangian are not typical of Lagrangians of the general Emden-Fowler equation. It is well-known that the cases $n=2$ and $n=-3$ differ from the general case 22]. Were we to use another value of $n$, the number of approximate 
symmetries would differ and the symmetries, $G_{7}$ and $G_{8}$, at the first order would not occur. We note that the Lie symmetry, $G_{2}$, of this Emden-Fowler equation is not a Noether symmetry.

\section{$4 \quad$ Lie integrals}

The attraction of Noether's Theorem is that the procedure for obtaining a first integral/invariant is trivial once the symmetry and gauge function have been determined, a process which is simultaneous. Of course there is a price to pay and that is the close relationship between the dependence of the coefficient functions on the derivatives and that of the integral/invariant 25]. One must look to generalized symmetries to be assured of finding the requisite number of invariants to know that a system is integrable. Apart from the practical problem of a more complicated calculation there is always the concern that one may have used the wrong Ansatz for the functional dependence on the derivatives in the coefficient functions. The Lie method is kinder in that respect, but the kindness is tempered with the additional burden of not completely friendly calculations. For multidimensional systems a combination of Noether's Theorem coupled with Lie symmetries has been found to be advantageous, particularly as, in the Lie approach, one can always insist that the integral be invariant under more than one symmetry [4].

We noted at the conclusion of the previous section that the Lie symmetry, $G_{2}$, listed in (2.9) is not a Noether symmetry and so does not have the easy route to an invariant which Noether's Theorem provides. We know from the existence of $I_{1}$ in (3.9) that the Emden-Fowler equation (2.7) is solvable as a consequence of Liouville's theorem 19. However, can we look to $G_{2}$ to provide an invariant which with $I_{1}$ will give the solution? For this we use the Lie method outlined in $\S 1$, 1.8). The invariants of $G_{2}=t \partial / \partial t-2 x \partial / \partial x$ are found from the solution of the associated Lagrange's system

$$
\frac{\mathrm{d} t}{t}=\frac{\mathrm{d} x}{-2 x}=\frac{\mathrm{d} \dot{x}}{-3 \dot{x}}
$$

and are

$$
u=x t^{2} \quad \text { and } \quad v=\dot{x} t^{3} \text {. }
$$

The second requirement of (1.8) is now expressed as

$$
\frac{\mathrm{d} I(u(t, x), v(t, \dot{x}))}{\mathrm{d} t}=0
$$

and leads to the second associated Lagrange's system,

$$
\frac{\mathrm{d} u}{v+2 u}=\frac{\mathrm{d} v}{\epsilon u^{2}+3 v}
$$

which is an Abel's equation of the second kind and, like most Abel's equations of either kind, is not responsive to known methods of integration. Consequently the existence of a second exact Lie point symmetry does not help in the search for a second integral. In terms of the conventional approach one is not surprised as the Lie Bracket of $G_{1}$ and $G_{2}$ is $\left[G_{1}, G_{2}\right]=G_{1}$ and we are effectively reducing the order by means of the nonnormal subgroup, $G_{2}$, which is well-known to remove $G_{1}$ as a point symmetry of the 
reduced equation. However, one may use an unconventional approach to attain our desired objective. In the reduction of order we know that the symmetry, $G_{1}$, becomes nonlocal. We know that the type of nonlocality is quite specific. The point symmetry becomes an exponential nonlocal symmetry and exponential nonlocal symmetries are known to be sufficient for the reduction of order [7]. This is not precisely what we wish to do here since the second order ordinary differential equation has already been reduced to a first order ordinary differential equation and the further reduction to an algebraic equation, while perhaps of some mathematical interest and pleasure, does not really further for the solution process. What we wish to do is to use the nonlocal symmetry to transform the first order ordinary differential equation to a form such that the solution of the first order ordinary differential equation is reduced to an elementary procedure. The reversion of the process of reduction of order to a first order ordinary differential equation which can be solved is not normally a trivial task. Here this is not a concern as we already have one first integral and that coupled with the invariant we seek now is all that is required. (Of course we know that the solution of this Emden-Fowler equation is expressed in terms of elliptic functions and so we do not expect to be able to write $x(t)$ in a simple form.)

The symmetry, $G_{1}$, becomes

$$
X_{1}=\exp \left[-\int \frac{\mathrm{d} u}{2 u+v}\right]\left(2 u \frac{\partial}{\partial u}+3 v \frac{\partial}{\partial v}\right)
$$

when we reduce the order of (2.7) to the first order ordinary differential equation (4.4). Were (4.5) a point symmetry we would seek a transformation which would make (4.4) autonomous by converting (4.5) to a simple $\partial / \partial X$. However, (4.5) is not a point transformation and we cannot expect to be able to achieve that much. We use a modification of the standard method which seeks a transformation [3, p. 110]

$$
X=F(u, v), \quad Y=G(u, v)
$$

so that the functions $F$ and $G$ satisfy the linear first order partial differential equations

$$
X_{1} F=1 \quad \text { and } \quad X_{1} G=0 .
$$

We simply replace the 1 in the first of (4.7) by the exponential part of the nonlocal symmetry (4.5), so that in the new coordinates $X_{1}$ will still be an exponential nonlocal symmetry of the form

$$
\bar{X}_{1}=\exp \left[-\int \frac{\mathrm{d} u}{2 u+v}\right] \frac{\partial}{\partial X} .
$$

Naturally the integral would be expressed in the new variables when the relationship is known. This stratagem means that the exponential term does not enter into any calculations. The characteristic for the independent variable is easily computed to be

$$
w=v u^{-\frac{3}{2}} .
$$

The other characteristic (for the first of (4.7)) requires the solution of

$$
0=\mathrm{d} F-\frac{\mathrm{d} u}{2 u}
$$


which very easily gives

$$
\zeta=F-\frac{1}{2} \log u \text {. }
$$

Under the change of variables

$$
X=v u^{-\frac{3}{2}}, \quad Y=\log u^{\frac{1}{2}}
$$

(4.4) takes the somewhat more friendly form

$$
0=\frac{\mathrm{d} Y}{\mathrm{~d} X}+\frac{X+2 \exp [-Y]}{3 X^{2}-2 \epsilon}
$$

which is linear in the variable $\exp [Y]$ and we immediately obtain the invariant

$$
J=\mathrm{e}^{Y} 3 X^{2}-2 \epsilon^{1 / 6}+2 \int\left(3 X^{2}-2 \epsilon\right)^{-5 / 6} \mathrm{~d} X .
$$

That the expression for $J$ is complicated is not surprising, but it is a precise form and maybe gives a different approach to the investigation of elliptic functions.

The case of the Emden-Fowler equation has not given much joy for the use of approximate symmetries to obtain approximate integrals/invariants as there are none which satisfy the criterion of not disappearing with increasing powers of the small parameter $\epsilon$. However, we can illustrate the way approximate symmetries can give truely approximate integrals/invariants with the simple harmonic oscillator. We should not be surprised at this for, if something does not work for the simple harmonic oscillator, it shall never work for anything. One regrets that there are some procedures which work only for the simple harmonic oscillator and that is the only model upon which trials are conducted!

\section{Discussion}

We have specified the approximate symmetries for the simple harmonic oscillator treated as a perturbation of the free particle, the Ermakov-Pinney equation treated as a perturbation of the equation for the simple harmonic oscillator and an autonomous Emden-Fowler equation of order two considered as a perturbation of the free particle. In the case of the simple harmonic oscillator the number of exact symmetries is eight. The number of approximate symmetries was found to be sixteen at $O(\epsilon)$, twenty-four at $O\left(\epsilon^{2}\right)$ and thirty-two at $O\left(\epsilon^{3}\right)$. The homogeneity symmetry $x \partial / \partial x$ occurs at all orders of $\epsilon$. The type of approximate symmetries that arise can be put into three categories: Those that are common to both perturbed and unperturbed equations; then the truely approximate symmetries the level of approximation of which increases with increasing powers of $\epsilon$. The third category consists of those symmetries in which every term is dependent on $\epsilon$. The Ermakov-Pinney equation has three exact symmetries instead of eight. When treated as a perturbation of the equation for the simple harmonic oscillator it has eleven approximate symmetries at $O(\epsilon)$, fourteen at $O\left(\epsilon^{2}\right)$ and seventeen at $O\left(\epsilon^{3}\right)$. The Ermakov-Pinney equation as a perturbation of the free particle has twelve approximate symmetries at $O(\epsilon)$, fourteen at $O\left(\epsilon^{2}\right)$ and sixteen at $O\left(\epsilon^{3}\right)$. Another aspect which has been investigated is that of Noether symmetries and approximate first integrals (or invariants). 
It is important to note that the knowledge of exact symmetries of a differential equation enables us to reduce it and, if possible, completely solve it to obtain exact solutions. However, if one seeks for approximate rather than exact solutions, then approximate symmetries are as useful as exact ones and can be determined perturbatively. In this paper we have emphasized more the study of approximate symmetries rather than approximate solutions. Finally we should note that in principle there exists the possibility of the intrusion of secular terms into the expressions for the approximate symmetries and integrals. This is not the case with the equations and integrals treated here. As applications of approximate symmetries have so far been at the first order in $\epsilon$, whence the motivation of this paper which was to look beyond the first order in $\epsilon$, there is no evidence that secular terms and lack of convergence would present a problem. The purpose of this paper was to demonstrate the different types of approximate symmetry available, a result which is not accessible when approximate symmetries are treated to the first order only as has been the case in the earlier literature.

\section{Acknowledgements}

PGLL thanks Professor G P Flessas, Dean of the School of Sciences, and Dr S Cotsakis, Director of GEODYSYC, for their kind hospitality while this work was undertaken and the National Research Foundation of South Africa and the University of Natal for a sabbatical grant.

\section{References}

[1] Baikov V A, Gazizov R K and Ibramigov N H, Approximate Symmetries of Equations with a Small Parameter, Mat. Sb., 1988, V.136, 435-450 (Math. USSR Sb., 1989, V.64, 427-441).

[2] Baikov V A, Gazizov R K and Ibramigov N H, Approximate Transformation Groups and Deformations of Symmetry Lie Algebras, in CRC Handbook of Lie Group Analysis of Differential Equations, Editor N H Ibragimov, CRC Press, Boca Raton, Florida, 1995, V.3, Ch.2.

[3] Bluman G W and Kumei S, Symmetries and Differential Equations, Appl. Math. Sci., Springer-Verlag, New York, 1989, V.81.

[4] Cotsakis S, Leach P G L and Pantazi H, Symmetries of Homogeneous Cosmologies, Grav. \& Cosmol., 1998, V.4, 314-325.

[5] Ermakov V, Second Order Differential Equations. Conditions of Complete Integrability, Univ. Izvestia Kiev, Ser. III, 1880, V.9, 1-25 (trans. A O Harin).

[6] Gazizov R K, Invariants of Approximate Transformation Groups, in Modern Group Analysis VI, Editors N H Ibragimov and F M Mahomed, New Age International Publishers, New Dehli, 1977, 149-158.

[7] Géronimi C, Feix M R and Leach P G L, Exponential Nonlocal Symmetries and Nonnormal Reduction of Order, Preprint: MAPMO, Département Mathématique, Université d'Orléans, Orléans la Source, 45067 Orléans Cedex 2, France, 1997.

[8] Govinder K S and Leach P G L, The Nature and Uses of Symmetries of Ordinary Differential Equations, S. Afr. J. Sci., 1996, V.92, 23-28.

[9] Govinder K S, Heil T G and Uzer T, Approximate Noether Symmetries, Phys. Lett. A, 1998, V.240, 127-131. 
[10] Head A K, LIE, a PC Program for Lie Analysis of Differential Equations, Comp. Phys. Comm., 1993, V.77, 241-248.

[11] Ibragimov N H, Elementary Lie Group Analysis and Ordinary Differential Equations, John Wiley \& Sons Ltd, Chichester, 1999, 231-234.

[12] Johnson R, Private Communication, 1979.

[13] Komar A, Asymptotic Covariant Conservation Laws for Gravitational Rediation, Phys. Rev., 1962, V.127, 1411-1418.

[14] Komar A, Positive-Definite Energy Density and Global Consequences for General Relativity, Phys. Rev., 1963, V.129, 1873-1876.

[15] Leach P G L, Cotsakis S and Flessas G P, Symmetry, Singularities and Integrability in Complex Dynamics I: The Reduction Problem, J. Nonlin. Math. Phys., 2000, V.7, N 4, 445479 .

[16] Leach P G L, Cotsakis S and Flessas G P, Symmetry, Singularities and Integrabily in Complex Dynamical Systems II: Rescaling and Time-Translation in Two-Dimensional Systems, J. Math. Anal. Appl. 2000, V.251, 587-608.

[17] Lie S, Differentialgleichungen, Teubner, Leipzig, 1891 (reprinted Chelsea, New York, 1967).

[18] Lie S, Berühungstransformationen, Teubner, Leipzig, 1896 (reprinted Chelsea, New York, 1977).

[19] Liouville J J, Sur l'intégration des équations différentielles de la Dynamique, J. Math. Pures Appl., 1855, V.20, 137-138.

[20] Mahomed F M and Leach P G L, Symmetry Lie Algebras of $n$th Order Ordinary Differential Equations, J. Math. Anal. Appl., 1990, V.151, 80-107.

[21] Matzner R A, 3-Sphere "Backgrounds" for the Space Sections of the Taub Cosmological Solution, J. Math. Phys., 1968, V.9, 1063-1066.

[22] Mellin C M, Mahomed F M and Leach P G L, Solutions of Generalised Emden-Fowler Equations with Two Symmetries, Int. J. Nonlin. Mech., 1994, V.29, 529-538.

[23] Noether E, Invariante Variationsprobleme, Koenig. Gess. Wissen. Nach. Math.-Phys. K, 1918, Heft 2, 235-269.

[24] Pinney E, The Nonlinear Differential Equation $y^{\prime \prime}(x)+p(x) y+c y^{-3}=0$, Proc. Amer. Math. Soc., 1950, V.1, 681.

[25] Sarlet W and Cantrijn F, Generalizations of Noether's Theorem in Classical Mechanics, SIAM Review, 1981, V.23, 467-494.

[26] Spero A and Baierlein R, Approximate Symmetry Groups of Inhomogeneous Metrics, J. Math. Phys., 1977, V.18, 1330-1340.

[27] Spero A and Baierlein R, Approximate Symmetry Groups of Inhomogeneous Metrics: Examples, J. Math. Phys., 1978, V.19, 1324-1334.

[28] Zalaletdinov R, Approximate Symmetries in General Relatvity, Preprint, International Center for Relativistic Astrophysics, Dipartamento di Fisica, Universitá di Roma "La Sapienza", P Aldo Moro 5, Roma 00185, Italy. 